Ondřej PEŠSK

Université de Bohême du Sud, České Budějovice

\title{
LA NORME ET LA DYNAMIQUE HISTORIQUES - LE CAS DE L'ACCORD DU PARTICIPE PASSE APRES LE VERBE AVOIR EN FRANÇAIS
}

\section{Objectif de l'étude}

Si nous allons maintenant consacrer quelques pages à la règle de l'accord du participe passé (PP) après le verbe avoir, ce n'est pas pour disserter encore une fois sur la complication dont ce vieil épouvantail des manuels scolaires peut se vanter ni pour apporter de nouvelles preuves et témoignages du non respect de cette norme de la part des Français contemporains (et anciens tout aussi bien). Car l'objectif de la présente étude est double: analyser d'abord les facteurs qui déterminaient dans le passé la dynamique relative aux réalisations de l'accord et ensuite démontrer quelles conséquences pour les locuteurs peut avoir une codification prématurée et trop dogmatique.

\section{Origines de la structure}

Regardons d'abord les origines de cette structure grammaticale. Comme le syntagme avoir + PP est un phénomène panroman, celles-ci remontent naturellement à la langue latine.

Le latin classique connaissait déjà les structures de type :

1. Satis habeo deliberatum (j'ai suffisamment délibéré) ou avec un COD :

2. Habeo epistulam scriptam, (j'ai écrit une lettre) structures qui se sont répandues en latin vulgaire et qui étaient à l'origine du passé composé des langues romanes. Il est évident que le verbe habeo a gardé ici son sens plein, de même que sa valence - epistulam est ici le COD du verbe habeo, le participe qualifie le COD et est régi par lui. Le verbe habere n'avait pas en latin classique de caractère auxiliaire et les structures de ce type ne pouvaient donc nullement être interprétées comme « temps composés » qui n'émergent, sur la base de ce type de structures, que plus tard dans le latin vulgaire. Il faut noter que la désémantisation du verbe habere est un processus très lent et ce n'est qu'au $\mathrm{VI}^{\mathrm{e}}$ siècle que les structures habere $+P P$ commencent à se multiplier. Dans les documents rédigés dans ce «latin vulgaire avancé » on peut trouver des phrases avec habere $+P P$ ou habere garde son sens plein à côté des phrases ou le verbe tend nettement à devenir simple auxiliaire :

3. Dotis quam promissam ab sponso habeo (la dot que j'ai promise par mon fiancé)

4. Promissum habemus ... nihil sine ejus consilio agere (Grégoire de Tours cité par BRUNOT, 1966 : I/87) (nous avons promis de ne rien faire sans son conseil)

\section{Ancien français}

La situation qu' on constate en ancien français (acception large - IX $-\mathrm{XIII}^{\mathrm{e}}$ siècles) témoigne encore une fois du fait que la grammaticalisation du verbe avoir s'est opérée lentement et dans un espace de temps très étendu ; cette évolution est liée à celle de la valeur du passé composé (et analogiquement des autres formes 
composées avec avoir): action accomplie dans le présent (valeur héréditaire, faible degré de grammaticalisation, soudure du participe et de l'auxiliaire très faible) $\rightarrow$ action accomplie dans le passé, équivalent du passé simple (nouvelle valeur émergente, grammaticalisation achevée, le participe et l'auxiliaire forment un tout inséparable), les valeurs nouvelles coexistant avec les valeurs héréditaires tout au long de la période de l'ancien français. La valeur de l'accompli résultatif dans le présent est bien observable dans le cas des verbes transitifs directs. Claude Buridant relève des exemples ou «le participe peut encore être senti comme un prédicat du régime avec un verbe avoir ayant encore son sémantisme plein » (BURIDANT, 2000: 376). Cette interprétation du statut du participe s'impose notamment quand le COD précède le participe. Cet ordre était particulièrement fréquent en ancien français :

5. Vos li avez tuz ses castels toluz... (Roland, 236) ${ }^{1}$

6. J'ai le François encore emprisonné... (Huon, 6409)

Le caractère résultatif de la construction auxiliaire + PP se manifeste clairement dans les emplois transitifs du verbe morir :

7...qui muert del mal don il l'a morte. (Cliges 4306)

Dans ces séquences, l'accord était plutôt de règle, même si on trouve aussi de nombreux contre-exemples, dus le plus souvent aux raisons métriques.

Dans les séquences où le COD suit le participe (Aux $-\mathrm{P}-\mathrm{O} ; \mathrm{P}-\mathrm{Aux}-\mathrm{O} ; \mathrm{P}$ - O - Aux), le participe reste invariable dans la majorité des cas :

8...messire Rogier avoit pris sa gent... (Morée, p.318) ${ }^{2}$

Cependant on rencontre dans les textes anciens des occurrences où le participe s'accorde avec le régime de la forme composée, essentiellement en poésie, les raisons métriques imposant la forme au participe :

9. Et l'empereres rot assemblees ses genz (Conqueste V, 451)

Pour résumer la situation en ancien français, on peut constater que :

1) si le COD (nominal ou pronominal) précède le participe, celui-ci s'accorde habituellement avec le COD. Dans certaines séquences de ce type, le verbe avoir s'est conservé un haut degré de sémantisme plein, le syntagme avoir + PP revêt la valeur de la structure primitive héritée du latin vulgaire;

2) si le participe suit le COD, il y a une forte tendance à l'invariabilité qui s'accentue au cours du XIII ${ }^{\mathrm{e}}$ siècle.

Dans les deux cas, maintes exceptions peuvent être relevées, mais il semble bien qu'elles soient dues essentiellement aux raisons métriques ou stylistiques.

On peut identifier trois facteurs majeurs (pas seuls, bien entendu) qui jouaient en ancien français en faveur de la réalisation de l'accord:

1) emploi très fréquent en ancien français des formes composées avec leur valeur héréditaire (accompli résultatif), le verbe avoir gardant un haut degré du sémantisme plein. Cette structure influait sur celles avec avoir auxiliaire pleinement grammaticalisé. La fréquence de l'emploi des formes composées ayant cette valeur montre qu'il s'agit d'une des valeurs centrales, au moins au début de l'histoire de la langue française ;

\footnotetext{
${ }^{1}$ Les exemples 5, 6, 7 et 9 sont cités d'après BuRIDANT, 2001: 376-378

${ }^{2}$ Exemple trouvé dans la Base de Français Médiéval (BFM)
} 
2) possibilité d'intercaler le COD nominal entre l'auxiliaire et le participe qui le suivait de près - la proximité de ces deux termes facilitait le phénomène d'accord. Sans être exclusivement réservée aux emplois héréditaires des formes composées, cette structure était prototypique pour la valeur de l'accompli résultatif au présent (valeur primitive des formes composées) ;

3) facteurs phonétiques - les $s$ finals se prononçaient, de même que tout les $e$ féminins. L'accord était bien pertinent même à l'oral.

On peut postuler que l'accord du PP en français résulte d'une certaine tension due à la coexistence au sein du système verbal de l'ancien français des valeurs héréditaires avec avoir non dématérialisé et des valeurs nouvelles avec avoir comme auxiliaire pleinement grammaticalisé. Si l'accord s'imposait naturellement dans le premier cas, on peut douter de son bien fondé dans le second, avoir et PP formant un syntagme bien soudé. Considéré de ce point de vue, l'accord du PP après avoir apparaît comme un résidu de l'ancien état de la langue. Le phénomène peut être analysé dans le cadre d'une dynamique déterminée par les paramètres linguistiques internes définis ci-dessus. L'évolution des valeurs des formes composées liée aux différents degrés de grammaticalisation du verbe avoir représente le facteur le plus important auquel s'ajoutent les deux autres. En fonction du statut de ces trois paramètres au cours du développement de la langue, l'accord du PP s'imposait d'une manière plus ou moins importante.

\section{Moyen français}

En moyen français, l'usage était flottant. Dans les textes, l'accord se faisait ou ne se faisait pas. Si dans l'ordre COD + participe, les copistes s'efforcent d'appliquer la règle de l'accord (mais souvent oublient de le faire), dans l'ordre participe + COD ils ont une forte tendance à laisser le participe invariable (et pourtant on relève de nombreuses occurrences de l'accord dans ce cas) ${ }^{3}$. Tout ceci montre la fragilité de l'accord dont la réalisation était sentie, semble-t-il, comme de moins en moins impérative (par rapport à l'ancien français).

\section{La « norme » de Marot}

$\mathrm{Au} \mathrm{XVI}^{\mathrm{e}}$ siècle, à la naissance de l'imprimerie, les premières tentatives normalisatrices apparaissent motivées par un souci d'améliorer la langue française, désormais langue officielle de l'état. Et c'est dans ce contexte que Clément Marot, dans son poème célèbre, pose la règle de l'accord du participe passé après le verbe avoir. Se référant à l'italien qui «passe les vulgaires du monde », il fixe le principe de la position du COD et ceci quelle que soit la valeur temporelle ou aspectuelle du syntagme avoir + PP. Il faut bien souligner qu'à l'époque où Marot l'édicte, cette règle n'avait en soi rien d'évident. D'abord, l'usage réel du XVI siècle était loin de la suivre, F. Brunot (1966 : II/468-470) cite bien des exemples où les bons auteurs contemporains de Marot ne l'observent nullement - soit ils font l'accord là où la règle l'interdit, soit ils ne le font pas là où elle le prescrit :

10. mignonne, allons voir si la rose Qui ce matin avait desclose sa robe de pourpre au soleil (Ronsard)

11. Les douleurs qu'en i'auray receu par vous (Ronsard)

\footnotetext{
${ }^{3}$ Marc WiLmet (1999 : 17) explique cette pratique par la « loi du moindre effort».
} 
Ensuite, cette règle, qui n'avait pas l'usage à son appui, ne faisait pas l'unanimité parmi les grammairiens contemporains de Marot. L'un des plus connus d'entre eux, Meigret qualifie les phrases de type 12. le graçes qe ie vous'ey fęttes de «lourdes incongruités reçúes pour courtisanes elegantes ».

Il est évident qu'il s'agit là du moment clé de toute l'histoire de la règle de l'accord. $\mathrm{Au}$ cours des $\mathrm{XV}^{\mathrm{e}}-\mathrm{XVI}^{\mathrm{e}}$ siècles les structures phrastiques françaises changent profondément se rapprochant nettement de la syntaxe du français moderne. L'emploi des formes composées dans leurs valeurs héréditaires, c'est-àdire accompli résultatif, si fréquent au moyen âge, va diminuant et devient secondaire. Nous pouvons caractériser cette époque comme une synchronie dynamique pendant laquelle dans l'usage oral du peuple et écrit des érudits coexistaient les valeurs anciennes avec les valeurs nouvelles prédominantes, mais une synchronie non encore ligotée par les prescriptions des grammairiens. Ce n'est qu'au cours du XVI $\mathrm{XI}^{\mathrm{e}}$ siècle que commencent à apparaître les premiers ouvrages cherchant à poser une norme aux usages, à codifier et unifier la langue (Meigret, Peletier, Ramus, H. Estienne, Abel Mathieu), efforts qui domineront les écrits linguistiques au siècle suivant. Le poème de Marot se situe évidemment dans ce contexte.

On a bien vu qu'en matière de l'accord du PP après le verbe avoir l'usage était bien flottant et très instable, toutefois la tendance à l'invariabilité était sensible. Les trois facteurs favorisant l'accord au Moyen âge ne se manifestaient plus avec la même pertinence :

1) la valeur principale du passé composé est celle de l'action accomplie dans le passé, quoique pas lointain (règle des 24 heures), le verbe avoir dans les formes composées étant senti comme un morphème purement auxiliaire. La valeur aspectuelle de l'accompli résultatif se situe plutôt à la périphérie ;

2) on constate pourtant une relative indépendance entre le participe et l'auxiliaire - de nombreux éléments dont le COD nominal pouvaient encore s'intercaler entre l'auxiliaire et le participe

13. «(François I) ... a nostre Langaige, au paravant scabreux \& mal poly, rendu elegant $\gg$ (Rabelais cité par Chaurand, 1999: 217).

L'ordre SVO s'impose, pourtant on peut encore rencontrer des séquences $\mathrm{OV}(\mathrm{S})$ le COD précédant le syntagme verbal :

14. m'amour vous ay donnée (Marot).

Ces structures deviennent agrammaticales aux cours du XVIII ${ }^{\mathrm{e}}$ siècle.

3) Les $s$ finals, marque du pluriel, ne se prononcent plus, de même que les $e$ féminins après voyelle. Dans ce cas ils produisent toutefois un effet phonétique prolongent la voyelle précédente, ce qui rend l'accord en genre dans la langue parlée plus pertinent que de nos jours. Cette pratique se maintient jusqu'aux environs du XVIII ${ }^{\mathrm{e}}$ siècle.

Nous voyons que cette situation est moins favorable à la réalisation spontanée de l'accord dans la langue parlée qu'en ancien et moyen français, mais toujours est-il que son maintien se justifie vu notamment les facteurs 2 et 3 .

\section{Splendeur et misère de la règle}

L'on connaît bien la direction que suivait l'évolution de la langue, tant au niveau des valeurs des formes composées qu'au niveau de l'ordre des constituants 
phrastiques et de la prononciation des $e$ féminins. L'on pourrait supposer que l'usage finirait par imposer l'invariabilité du participe dans toutes les positions, comme c'est le cas de l'espagnol, du portugais, du provençal ou du roumain, n'était cette intervention extérieure qui, dans l'intention de mettre de l'ordre dans un usage flottant, a freiné la dynamique naturelle du système.

$\mathrm{La}$ règle posée par Marot est par la suite devenue comme le dit Brunot : «l'évangile des nouveaux grammairiens, un évangile sur lequel chacun raffine, subtilise et qui va bientôt servir de thème à une suite sans fin de disputes scolastiques » (BRUNOT, 1966 : III/601). De Vaugelas à Ménage, chacun traite de cet écueil de la grammaire en soulignant à la fois son importance et l'ignorance générale de cette règle de la part des usagers. Et malgré la véhémence avec laquelle les grammairiens insistent sur cette règle, les auteurs, même les «bons » pêchent contre elle constamment, ce qui en prouve encore une fois la fragilité déjà à l'époque où les facteurs syntaxiques et phonétiques pouvaient encore jouer en sa faveur. Nous n'allons pas bien entendu présenter ici toutes les subtilités de cette règle discutées par les grammairiens dans leurs écrits et dont l'héritage continue toujours à hanter l'enseignement scolaire. Nous allons juste consacrer un bref commentaire aux raisons qu'on avance à l'époque pour expliquer la nécessité d'accorder le participe. Là où le sentiment linguistique naturel des usagers se rebelle, les auteurs de la Grammaire générale, conformément à leurs objectifs, cherchent à démontrer la logique qui soutient cet accord. Pour ce faire, ils invoquent les structures latines du type quam habeo amatam en prétendant qu'elle correspond à la structure français que j'ai aimée - analogie évidemment fausse, parce que ce serait nier le statut d'auxiliaire qu'a le verbe avoir en français et qu'il n'a jamais eu en latin classique. Mais vu l'autorité dont jouissait la Grammaire générale à son époque, le problème du participe passé semblait résolu une fois pour toutes et toute remise en question de cette règle était vouée à l'échec.

Les conséquences de cette intervention normative des grammairiens, reposant souvent comme on vient de le voir, sur un raisonnement assez arbitraire, sont évidents. La norme a en effet généralisé une pratique qui n'était fondée que sur une valeur périphérique du syntagme avoir + PP. Jamais les locuteurs n'ont intériorisé cette règle, compliquée qu'elle est devenue par des amendements interminables allant parfois l'un à l'encontre de l'autre. Devant l'obligation ou non d'accorder le participe dont l'existence n'est relevée aux Français qu'à l'école, beaucoup se sentent en insécurité linguistique. Depuis qu'on a formulé la règle censée devenir norme jusqu'à nos jours, les locuteurs, y compris les bons écrivains (même très bons), ne cessent de pêcher contre elle. La règle reste pourtant sacrée : une tentative en 1900 de permettre le non-accord du participe passé avec avoir dans tous les cas (arrêté de Georges Leygues) a été immédiatement dénoncée par l'Académie française et l'arrêté a été remplacé par un autre qui ne réduisait la tolérance du non-accord qu'à de rares cas particuliers (arrêté du 26 février 1901 relatif à la simplification de l'enseignement de la syntaxe française). Deux autres actes législatifs tentant d'alléger la rigueur de la règle de l'accord ont été publiés au cours du XX ${ }^{\mathrm{e}}$ siècle - l'arrêté Haby en 1976 et la réforme de l'orthographe en 1990. Ni l'un ni l'autre n'osent aller très loin dans leurs recommandations, ne traitant que les cas où le participe est suivi d'un infinitif. 


\section{La situation contemporaine}

Bien des linguistes décrivant l'usage contemporain ne manquent pas d'affirmer qu'à l'oral, les Français ne font plus l'accord après le verbe avoir (ce qui d'ailleurs était souvent le cas depuis que la norme existe) et ceci quels que soient leur niveau d'études et la situation de communication (de l'ouvrier lillois causant avec ses potes jusqu'au président de la République s'adressant à la nation dans un discours télévisé). Mais pour analyser la situation contemporaine, nous croyons qu'il n'est pas possible d'y voir une dynamique qui se manifesterait dans les usages relatifs à l'accord du PP après le verbe avoir. Tous les paramètres linguistiques internes qui pouvaient favoriser la réalisation de l'accord, résidu, comme nous l'avons postulé, de la tension qui existait au sein du système de la LF au cours de l'évolution des valeurs des structures avec avoir auxiliaire, semblent être nuls en français contemporain :

1) en français contemporain, le PP forme avec l'auxiliaire un ensemble soudé. L'ancienne valeur héréditaire se perpétue dans les emplois où la valeur aspectuelle de ce tiroir verbal est mise en avant. Mais, contrairement à l'ancien français, il n'y a plus aucune hésitation relative au statut du verbe avoir qui a un rôle purement auxiliaire ;

2) le COD nominal ne peut jamais se placer entre l'auxiliaire et le participe structure qui grâce à la contiguïté du COD et du PP, favorise le déclenchement de l'accord (disparition de cette structure au cours du XVIII e siècle). S'il en est ainsi l'interprétation de la séquence est attributive, sans aucune équivoque :

15. Les maires ont des choses détruites (par les jeunes dans les banlieues depuis 1 an $)^{4}$;

la phrase suivante

16. *Les jeunes leur ont des choses détruites.

étant aujourd'hui agrammaticale ;

3) l'accord à l'oral ne concerne que quelques verbes, quoique fréquents, mais dans la plupart des cas il est phonétiquement non pertinent (la prolongation de la voyelle due au $e$ féminin disparaît au cours du XVIII ${ }^{\mathrm{e}}$ siècle).

En considérant ces constatations, nous sommes persuadé que l'accord du PP après le verbe avoir n'est plus structurellement fondé en français contemporain. Si encore aux $\mathrm{XVII}^{\mathrm{e}}$ et $\mathrm{XVIII}{ }^{\mathrm{e}}$ siècles des éléments se situant à la périphérie du système de la langue pouvaient en motiver les réalisations (étant donné le statut des trois paramètres définis ci-dessus), de nos jours il n'en reste plus de traces. L'accord ne se maintient que grâce à la pression de la norme enseignée à l'école et qui se superpose à l'usage spontané. Nous croyons que c'est aussi cette influence de la norme enseignée qui est à l'origine des réalisations des accords spontanées à l'oral que constate une étude publiée par Blanche-Benveniste et alii (1990). Les tendances relevées correspondent sans aucun doute à la réalité, mais il est difficile d'évaluer dans quelle mesure elles sont dues à un usage infléchi s'efforçant depuis des siècles de se plier aux prescriptions des grammairiens.

\footnotetext{
${ }^{4}$ A partir de la phrase entendue à la radio: Jean-Paul Huchon, France-Inter (L'invité de...), le 29 octobre 2006 :

«J'ai voté un fonds de 20000000 EUR pour indemniser les maires des banlieues qui avaient des choses détruites »
} 
Nous sommes persuadé (et ceci n'est que notre humble avis personnel) qu'il serait utile de ne plus insister sur le maintien de cette règle. Mis à part les raisons évidentes dénonçant le caractère passablement artificiel et hypercompliqué de la règle, l'abolition de l'accord après le verbe avoir pourrait aider à désambiguïser les structures dans lesquelles le participe fait parti intégrante de la forme verbale (ex. 18) et les structures dans lesquelles il est attribut du COD du verbe avoir qui, paraphrasable par posséder, garde pleinement son sémantisme et sa valence (ex. 17).

17. Les choses que les maires ont détruites (par les jeunes dans les banlieues depuis 1 an).

18. Les choses que les jeunes ont détruit (il y a un an).

Quoi qu'il en soit, la règle reste bel et bien partie intégrante de la norme prescriptive et elle le restera encore longtemps vu l'importance que les Français accordent aux normes de leur langue. Ce qui est une fois sacré par les institutions respectables a un avenir assuré. Le prestige de l'institution fonde le prestige des usages. En respectant la norme presriptive, et la règle de l'accord du PP en particulier, les locuteurs étalent leur culture, leur niveau d'études et leur prétendue supériorité sociale. Qui a une fois appris à maîtriser ces règles ne tolère pas qu'on ose lui ôter ce privilège : en abolissant les règles, on rend vain l'effort qu'il a dû faire pour les apprendre. Et ceci ne se pardonne que difficilement.

Nous devons donc nous plier à la réalité et continuer à apprendre la règle de l'accord. Nous autres professeurs de FLE aurons du moins de la matière à enseigner et des raisons de plus pour sanctionner les mauvais élèves.

\section{BIBLIOGRAPHIE}

BRUNOT, Ferdinand (1966), l'Histoire de la langue française des origines à nos jours, Volume I, II, II, IV, Paris, Armand Colin.

BURIDANT, Claude (2000), Grammaire nouvelle de l'ancien français, Paris, Sedes.

BLANCHE-BENVENISTE, Claire (1990), Le français parlé. Études grammaticales, Paris, Ed. du CNRS.

BMF: Corpus électronique : Base de Français Médiéval, http://bfm.ens-lsh.fr/.

CHAURAND, Jacques (1999), Nouvelle histoire de la langue française. Paris, Le Grand livre du mois.

WILMET, Marc (1999), Le participe passé autrement, Duculot, Paris, Bruxelles. 


\section{ABSTRACT}

The aim of this paper is to analyze the rule of past participle agreement during the evolution of the French language. Our interest is not to dissert on the complication of the rule but to bring out the factors determining the dynamics of the realization of this agreement in the past. We try to show that these factors are of semantic, syntactic and phonetic nature. Moreover, the rule is an excellent example which allows us to demonstrate the consequences that a mature codification can have for the evolution of a language. 